\title{
Energy absorption of aluminum panels subjected to gelatin projectile impact
}

D. $\mathbf{L i}^{\mathrm{a}}$

\section{W. Zhang ${ }^{\mathrm{a}^{*}}$}

L. Guo ${ }^{a}$

\section{W. Xie}

\section{Jiang ${ }^{a}$}

a Hypervelocity Impact Research Center, Harbin Institute of Technology, Harbin 150036, P.R. China. E-mail: leodart@126.com, zhdawei@hit.edu.cn, guolc@hit.edu.cn,675849319@qq.com, 1962564675@qq.com

*Corresponding author

http://dx.doi.org/10.1590/1679-78255604

\begin{abstract}
Digital image correlation (DIC) method has been widely used on dynamical experiments. This full-field and real-time method can fill in the gap of traditional point-based measurements of typical structures subjected to soft body impact such as bird strike. In order to get further understandings of soft body impact process, the present study analyzed the time-dependent energy exchange during impact experiments. The dynamic responses of the aluminum target panels were obtained using 3D digital image correlation method so that their displacement and strain field histories can be tracked. By introducing the material properties of the targets, their time-dependent stress state and consequently the strain energy can be calculated. With the help of time-dependent profiles of target panels, their energy absorption properties were theoretically analyzed, including the exchange of kinetic energy and plastic work. The results showed that when the impact loadings were increased, the plastic work generated by radial membrane force became the major source of energy dissipation. The transverse movements consumed more kinetic energies than rotatory moments. This research may provide a further application of DIC results and help to better understand the soft body impact process on targets with large deformations.
\end{abstract}

\section{Key words}

Gelatin impact; Gas gun system; Digital image correlations; Time-dependent deformations; Energy exchange; 


\section{Introduction}

Soft body impact, defined as impact events where the strength of projectiles is much less than the shock pressures, are of great concerns in aerospace engineering industries. Collisions during flight can cause fatal accidents, and $90 \%$ of them can be attributed to bird strike (Meguid et al., 2008a), which belongs to soft body impact issues. So far, extensive researches have been focused on estimating the impact resistance of common airplane structures, and systematic methodologies have been constructed.

In order to develop reliable experimental methods for bird strike researches, standard and repeatable bird projectiles were required. Real birds were not suitable to be used as projectiles due to their irregular shapes and structures. Wilbeck (1978); Wilbeck and Rand (1981) used a compressed air launching facility to conduct the bird strike experiments, and a Hopkinson bar were employed to measure the shock force. He found that gelatin projectiles with $10 \%$ porosity performed good consistency with real birds. Although this substitutive bird could not perfectly match the mechanical characteristics of real birds, the bird strike loadings were reproduced with good repeatability and operability. Based on the experiment results, the shock forces were much greater than the strength of the projectiles, which failed and became fluid-like rapidly after the impact happened. Therefore hydrodynamic theories were applied to the impact process, dividing it into four main regimes: (I) Initial shock phase, (II) Impact pressure decay, (III) Steady flow and (IV) Flow termination.

Until now, two types of projectiles are employed in bird strike experiments: dead birds and gelatin. Since the recipes of gelatin projectiles varied between different laboratories and are mostly unpublicized, Lavoie et al. (2009) developed the production steps of gelatin specimens as a reference of bird strike experiments and an estimation of its numerical models. The projectiles were launched on a rigid target where several carbon gages were attached to measure the pressures. The snap shots captured by a high-speed camera showed that they behaved hydrodynamically during impact, but their back surface slowed down as the impact proceeded. This phenomenon was inconsistent with Wilbeck's theory, where the impact has no effect on the velocity of projectiles' end part. Also, the pressure obtained by carbon gages were much higher than theoretical values. It can be seen from the literatures that the recipes of the gelatin projectiles are still not standardized. Shapes, densities and hydrodynamic behaviors are evaluated to ensure the effectiveness of the recipes (Anghileri et al., 2012; Corporation, 2012; Heimbs, 2011; Heimbs and Bergmann, 2012; Lavoie et al., 2007a; Lavoie et al., 2007b; Lavoie et al., 2009; Seidt et al., 2012). Liu et al. (2014) used dead chickens instead of gelatin to conduct the birdstrike experiments. Three constitutive models were employed to describe the dynamic behaviors of the birds for different loading conditions. By comparing the results of experiments and simulations, it can be seen that changing the models of the birds may result in different loading histories, and appropriate choices should be made according to the loading conditions. Another research using real birds projectiles was from Hanssen et al. (2006), who used a polynomial equation of state to model the bird projectiles. The local failure on the sandwich target were successfully described, but the strain histories only matched the first millisecond of the experiment results for low-density specimens. The author attributed this phenomenon to different shapes between real bird projectiles and ideal bird models used in the simulations, but further certifications were lacked.

Once the constitutive model of bird projectiles is determined, the calibrations of the parameters are conducted by comparing with the experimental results. Airoldi and Cacchione (2006) used Wilbeck's results to validate his model, where pressure-history data were compared. As Wilbeck's experiments were limited by backward test devices, and the working conditions were also uncleared, large uncertainties were brought to the evaluations. His work can be better regarded as a reference for general behaviors rather than quantitative criteria (Heimbs, 2011; Heimbs and Bergmann, 2012; Lavoie et al., 2007a; Lavoie et al., 2007b). Other literatures using existing experimental results as validation tools can be found in Mao et al. (2007); Mao et al. (2008); Mao et al. (2009); Meguid et al. (2008b); Nishikawa et al. (2011). Another way to evaluate the model is the parameter studies based on experimental and simulation results. Jeremy Seidt 
et al. (2012) employed a tube sensor to obtain the impact force of the gelatin projectiles. The basic profiles and the magnitudes of the data were consistent to the simulation results. A more direct way can be found in Liu et al. (2014), where Liu employed a Hopkinson bar to obtain the constitutive parameters of gelatin material. Strain gauges, laser sensors and load cells were used to get the response of targets.

Although extensive researches have been carried out to study the dynamic behavior of typical structures subjected to bird strike, the modeling method of bird is still not standardized. The evaluation of bird models is limited by data collection methods, which are usually point-based such as data from strain gauges or load cells. However, these results are not able to describe the dynamically distributed loading conditions of fluid-like projectiles, especially for targets with large deformations during impact. On the other hand, errors may arise from derivations between test sensors and data collections in the numeric models. Strain gauges tend to debond from some type of specimens such as carbon fiber reinforced composites. Moreover, the loading conditions may affect the accuracies of the contact sensors, such as temperature effect in the strain gauges and the aluminum Hopkinson bars (Chen and Song, 2011). Therefore, the motivation of the present work is to record the dynamic responses of targets with a full-field and non-contact technique, and to develop a more comprehensive method to evaluate the numeric models. Considering this, the 3D digital image correlation (DIC) method is introduced. Since introduced and developed at the University of South Carolina (Chu et al., 1985; Sutton et al., 1986; Sutton et al., 1983), DIC have become an established method and are widely used to measure full-field displacements of targets in mechanic experiments.

DIC is an optical measurement method using correlation algorithms to track the displacement histories of speckled objects (Chu et al., 1985). Reflection lights are the signal source of this technique, which are insensitive to environmental influences. Usually high-speed cameras are employed to obtain real-time digital images of targets during dynamic experiments. For impact issues, challenges lie on the adjustments of experiment conditions such as camera settings and light sources. For example, as a trade-off exists between the frame rate (frames per second) and the resolution of the image, they should be balanced to satisfy the requirements of both the continuity of impact process and the quality of images. Nowadays, DIC has been playing an important role in relatively extensive researches addressing high-strain-rate problems. Researchers tend to use this method to measure out-of-plane deflection as well as in-plane strain on metal or composite targets subjected to blast loading (Kumar et al., 2012; Tiwari et al., 2009) and drop-weight-impact loading (Bhatnagar, 2014; Flores et al., 2017; Lall et al., 2009; Tuo et al., 2019; Yuan and Wang, 2019; Zhang and Porfiri, 2019). The frame rates of the cameras used in these studies were set from 48,00 fps to 50,000 fps depending on different experimental demands. In his experimental and numerical work on hail stone impact, Tang et al. (2017) used DIC results to validate the constitutive model of ice material. In (Huang et al., 2018) and (Hild et al., 2019), DIC was employed to measure the deformation field of specimen in Hopkinson bar tests.

The advantages of DIC method against conventional point-based measurements can significantly expand its application. However, the use of DIC has only been focused on the illustration of experimental procedures. In this paper, further utilization of DIC results was developed to study the dynamic behavior of target panels subjected to gelatin impact. Strain energies in the panels were deduced from the 3D deformation histories using energy balance equations. For the consideration of simplifying calculations, isotropic aluminum alloy panels were used in the experiments. Different types of energy dissipations were analyzed, including kinetic energies and plastic energies induced by membrane strain and bending strains. This research may shed light on the methodology to make full use of DIC results in understanding material's dynamic mechanisms. 


\section{Experiment}

\subsection{Gas gun system}

The experiments were conducted in High Velocity Impact Dynamics Lab in Harbin Institute of Technology. A gas gun which can accelerate the projectiles to target velocities by a sudden release of pressurized gas was employed to launch the gelatin projectiles. Figure 1(a) shows the schematic of the gas gun system. The black narrows indicate the launch directions. The projectiles, carried by their sabots (Figure 1f), were pushed by high-pressure air through a three-meter-long gun barrel (Figure 1d). After the sabots were separated by a steel stripper (Figure 1e), the projectiles impacted on the target panels. Figure $1 \mathrm{c}$ shows a target panel and the cover plate. In this paper, the panels were supported by a 20-mm-thick steel plate and covered by a 10-mm-thick steel plate. Both the support plate and the cover plate have a 150-mm-diameter hole in them and were tightly screwed by eight circumferentially equispaced bolts, providing an approximately fixed boundary condition and a circle loading area. Figure $1 \mathrm{c}$ shows the fixture configuration where a steel rod was used for trajectory correction.
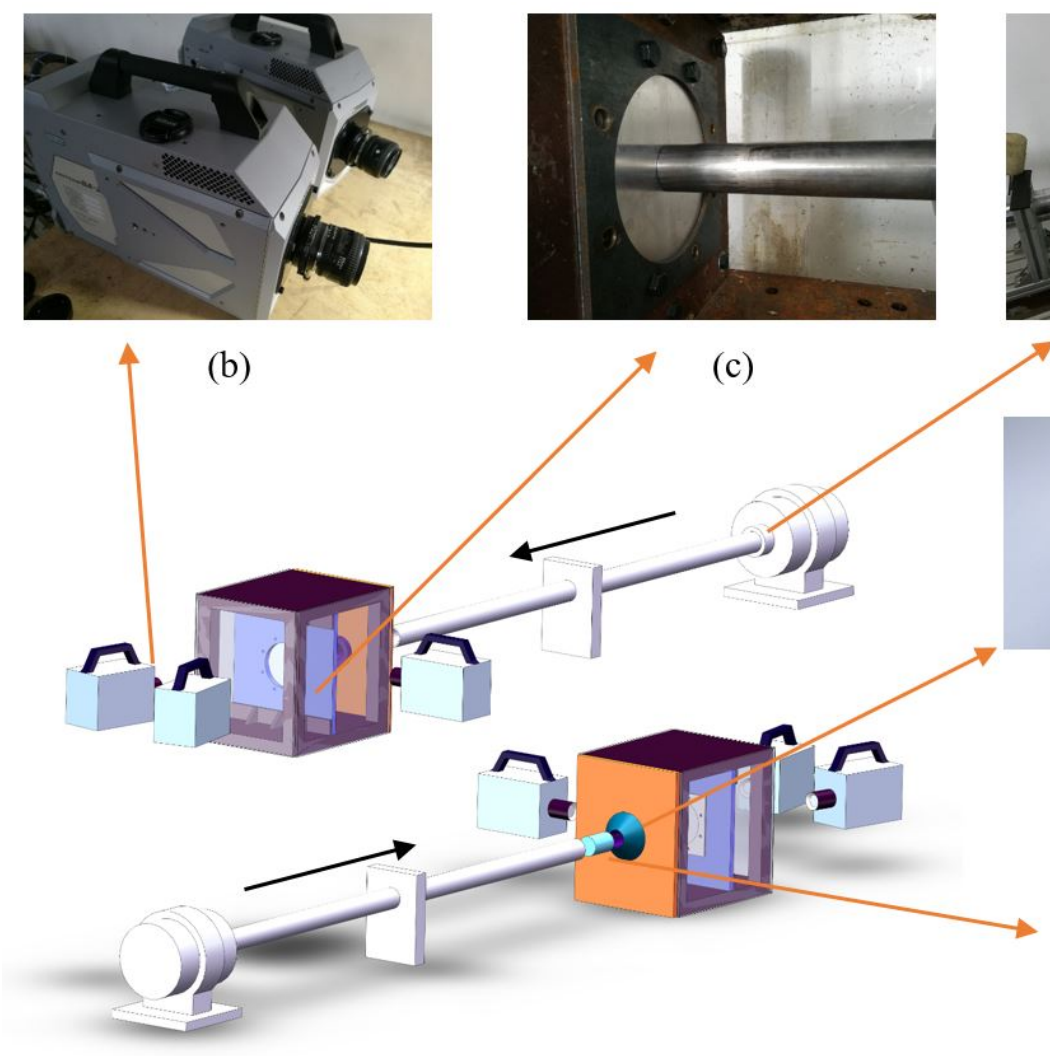

(a)

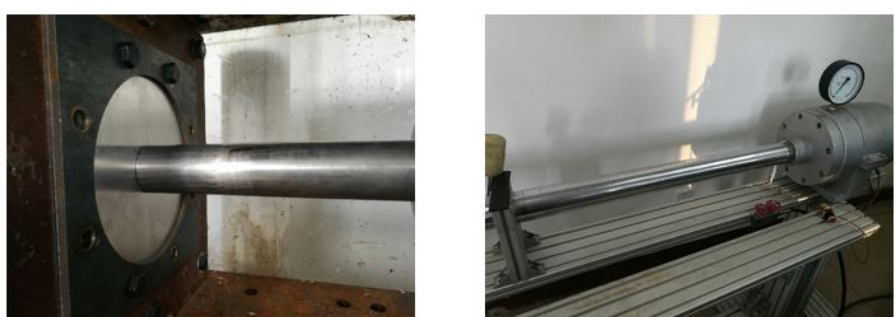

(d)

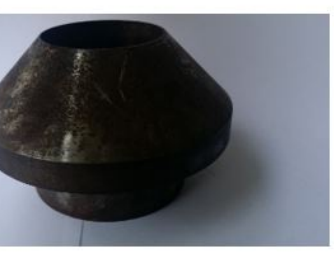

(e)

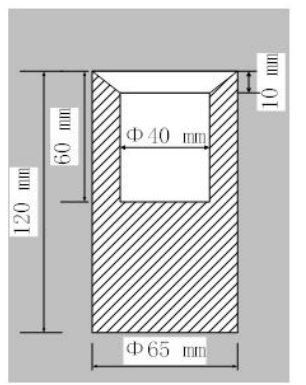

(f)

Figure 1. (a) Gas gun facilities; (b) High speed cameras; (c) Target panel and cover plate; (d) Single-stage gas gun; (e) Sabot striper; (f) Sabot

Two Photron SA-Z high speed cameras were placed about one meter away behind the targets to capture digital images for DIC calculations (Figure 1b). The placements of them were set according to the requirement of accurate DIC calculations. The angle between them was adjusted to $17.8^{\circ}$. The resolutions were set to 7680888 and the shutter time were $1 / 31520 \mathrm{~s}$. The frame rate, defined as numbers of pictures per second, were set to 30000 . These setups, along with high-strength cold light source, were modified to obtain clear shots of speckled panels without sacrificing too much 
brightness. GOM ARAMIS software was employed in this paper to calibrate the setups and process the DIC result. An $1750140 \mathrm{~mm}$ square GOM calibration board was used to calibrating the cameras, with a deviation of 0.074 pixels.

The velocities of projectiles were measured by another camera placed vertically beside the target box, with which the impact scenes can also be recorded (Figure 1). The scale factor of the real path length and the pixel length was calibrated by comparing the pixel length $l_{c}$ and the real length $l_{0}$ of a reference rod as Figure 2 shows, so that the real flying distances of the projectiles could be obtained from the camera. The velocities $v_{p}$ can therefore be calculated by:

$v_{p}=\frac{d_{p} l_{c}}{l_{0} t_{p}}$

where $d_{p}$ is the pixel distance the projectile travelled in recording duration $t_{p}$. The frame rate, shutter time and the resolution of this camera were set to $30000,1 / 7000 \mathrm{~s}$ and 6400376 respectively. This camera used the image trigger to generate trigger signals, which is available in Photron FASTCAM Viewer. When a selected area on the vision, specifically the trajectory zone, detected a sudden change of its gray level such as the flying projectiles, the software would send a trigger signal to the camera and started recording. The output channel of this camera was connected to the input trigger channels of the other two cameras on the backside with two wires of a same length. Once this camera was triggered, it sent a trigger signal to other two cameras. Using this trigging method, the recording was synchronized to ensure that the correlated images were captured at the same time.

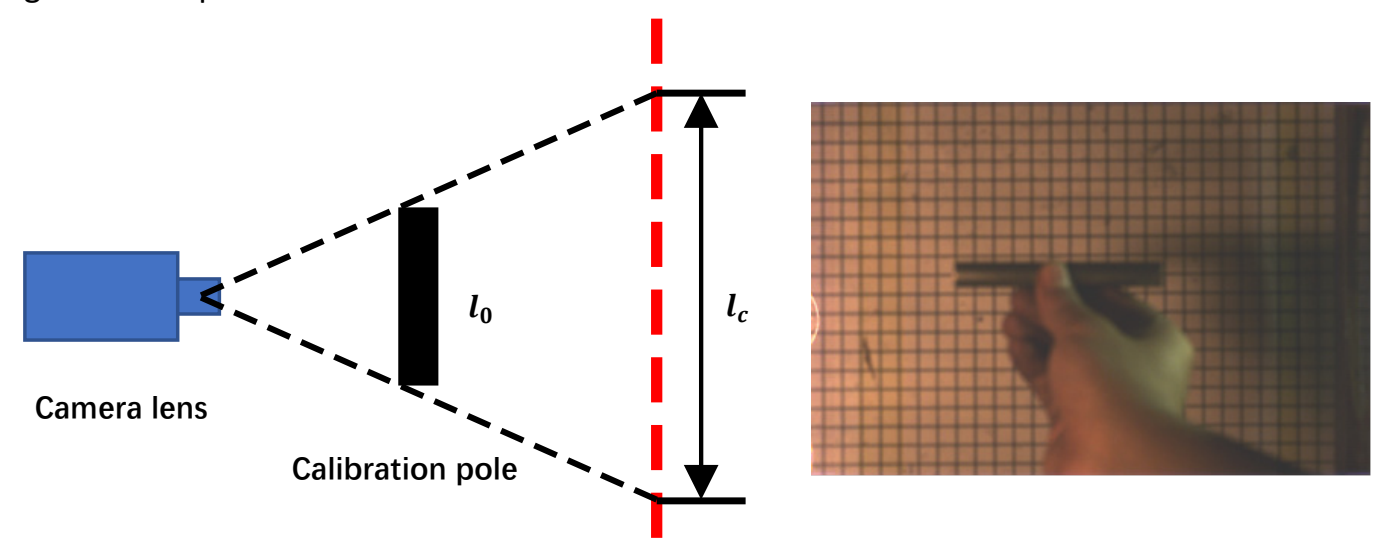

\section{Coordinate paper}

Figure 2. Velocities calibration method

\subsection{Target panels}

The target panels used in this paper were 2-mm-thick 2A12 T4 aluminum alloy plates with a size of $200 \mathrm{~mm}$ square. As mentioned before, the targets were mounted between a support plate and a cover plate, leaving only $150 \mathrm{~mm}$ diameter circular loading areas. The material parameters of 2A12 alloy were related to authors' previous work (Zhang et al., 2013). The material is described by a modified Johnson-Cook constitutive model, which is a piecewise equation based on necking effect: 


$$
\left\{\begin{array}{r}
\sigma_{e q}=\sigma_{s}+c_{2}\left[1-\exp \left(-\frac{\varepsilon_{e q}}{c_{1}}\right)\right], \varepsilon_{e q}<\varepsilon_{u}-\sigma_{s} / E \\
\sigma_{e q}=\sigma_{u}\left[\omega\left(1+\varepsilon_{e q}-\varepsilon_{u}+\frac{\sigma_{s}}{E}\right)+(1-\omega)\left(\frac{\varepsilon_{e q}}{\varepsilon_{u}}\right)^{\varepsilon_{u}}\right], \varepsilon_{e q} \geq \varepsilon_{u}-\sigma_{s} / E
\end{array}\right.
$$

Where $\sigma_{e q}$ and $\varepsilon_{e q}$ are equivalent stress and strain; $\sigma_{s}$ is the yield strength under reference strain rate and temperature; $\sigma_{u}$ and $\varepsilon_{u}$ are real stress and strain when necking; $c_{1}$ and $c_{2}$ are constant values; $\mathrm{E}$ is the young's modulus; $\omega$ is a weight parameter between 0 and 1 . All the parameters are shown in Table 1 . Figure 3 presents the load-displacement curves of 2A12 at different temperatures.

Table 1. Constitutive parameters of 2A12-T4

\begin{tabular}{|c|c|c|c|c|c|c|}
\hline$E / G P a$ & $\mathrm{~b}_{\mathrm{s}} / \mathrm{MPa}$ & $\mathrm{b}_{\mathrm{u}} / \mathrm{MPa}$ & $\omega_{u}$ & $C_{1}$ & $\mathrm{C}_{2} / \mathrm{MPa}$ & $\omega$ \\
\hline 71.7 & 400.0 & 635.0 & 0.1255 & 0.0713 & 288.0 & 0.0 \\
\hline
\end{tabular}

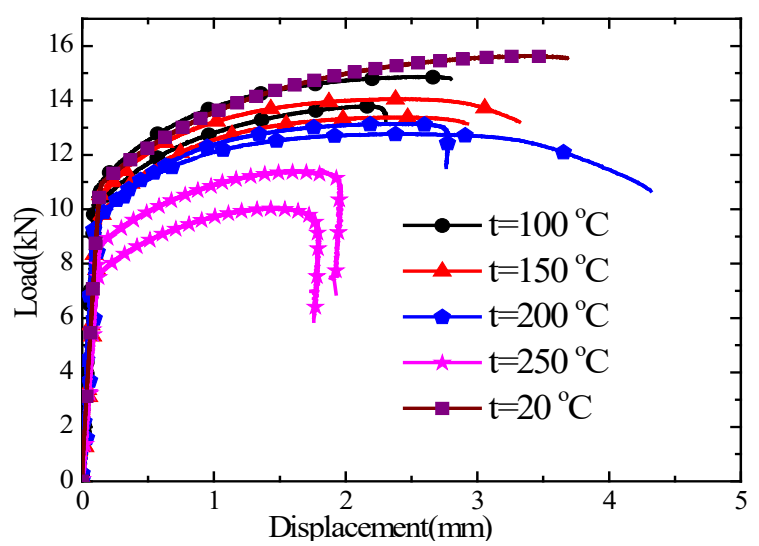

Figure 3. Load-displacement curves of $2 \mathrm{~A} 12$ at various temperatures

\subsection{Projectiles}

Gelatin projectiles are widely accepted to be used as substitutions of biological tissues, such as bird projectiles in this paper. When the impact happens, the particles on the front end of the bird are immediately brought to nearly static, and a compressive shock on the interface is generated between the target and the bird. When this shock meets the free surface of the bird, the unloading tensile wave can be formed. It is believed that this tensile stress is greatly larger than the strength of the bird, making it flow on the target. Among the tested low-strength materials, gelatin projectiles can mostly fit the flow properties and the impact loading histories of real birds. This make gelatin the best choice to conduct bird strike experiments.

In this paper, circular cylinder gelatin projectiles with a length of $80 \mathrm{~mm}$ and a diameter of $40 \mathrm{~mm}$ were used as soft impactors. Lavoie's recipe (Lavoie et al., 2009) was employed for making the projectiles. Figure 4 shows a gelatin projectile used in this research. All the specimens have a density around $950 \mathrm{~kg} / \mathrm{m}^{3}$. As mentioned before, foam-made sabots were used to carry the projectiles before they entered the target chamber. These sabots can protect the projectiles from getting deformed or off-course during accelerations. 


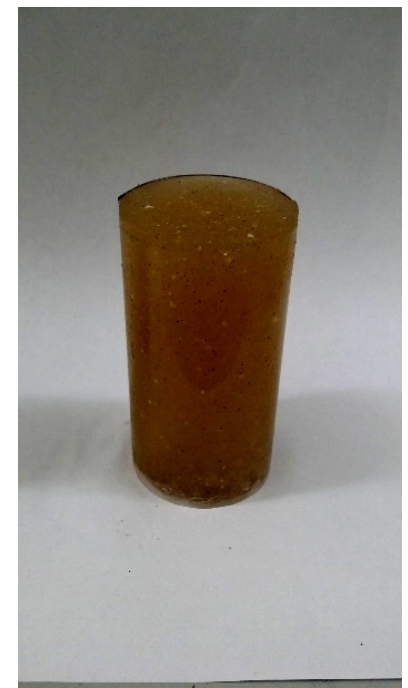

Figure 4. Gelatin projectiles

\section{Experiment results}

Before the experiment, one camera was placed on side of the target box and shoot a target panel with an oblique vision. Figure 5 illustrate a typical impact scene of the projectile, with an impact velocity of $69 \mathrm{~m} / \mathrm{s}$. As mentioned above, the radial tensile stress generated from the shock pulled the particles on the cylindric surface away from the center point and make them flow on the target panel. Based on equation (1), the impact duration is expected to be $1.16 \mathrm{~ms}$, which perfectly matches the results.

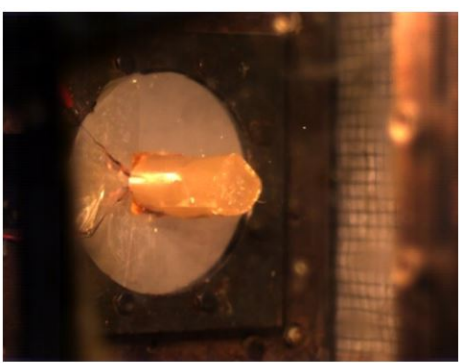

$0 \mathrm{~ms}$

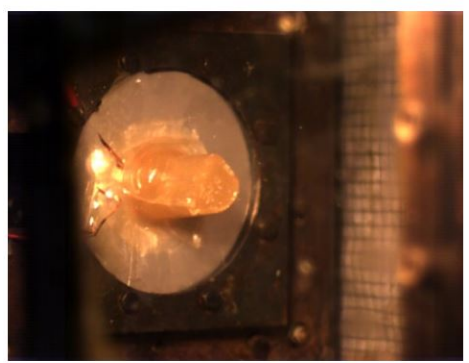

$0.6 \mathrm{~ms}$

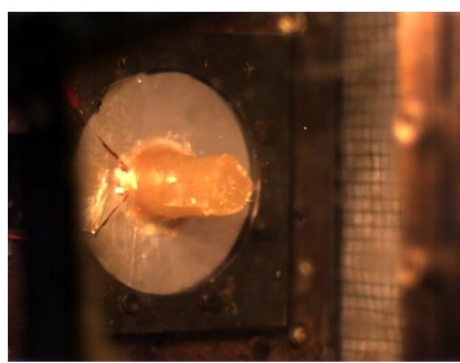

$0.2 \mathrm{~ms}$

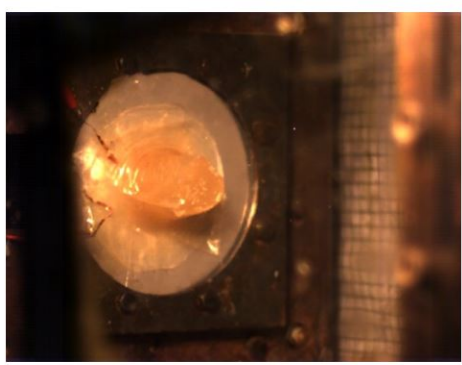

$0.8 \mathrm{~ms}$

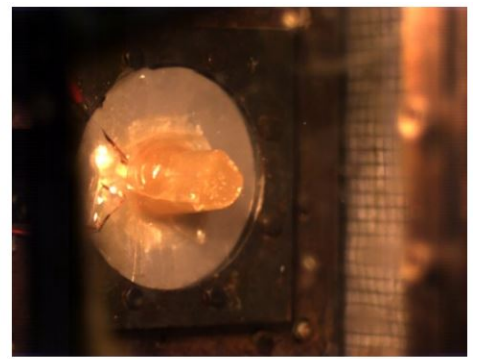

$0.4 \mathrm{~ms}$

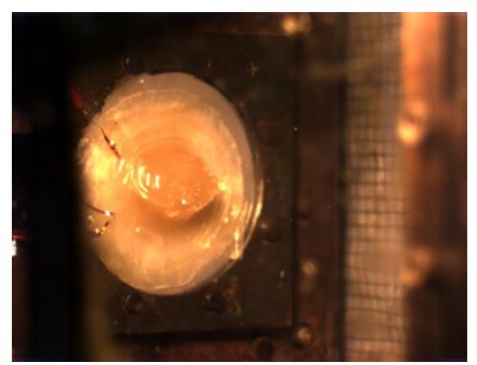

$1 \mathrm{~ms}$

Figure 5. Flowing process of projectile during impact

There are several treatments on DIC results since they are the major database of this experimental work. Firstly, to simplify the analysis, transformations of coordinates were conducted in Aramis software to confirm that the X-Y plane were located on the target panels, and the $Z$ axis were perpendicular to the target panels. Secondly, one spot on the 
support plate were also picked out to calculate its displacement, shown in Figure 6. These can be seen to be the rigid displacements during impact which were not caused by impact events, but from other extra factors such as vibrations of the test bed. Therefore they should be removed from DIC results.

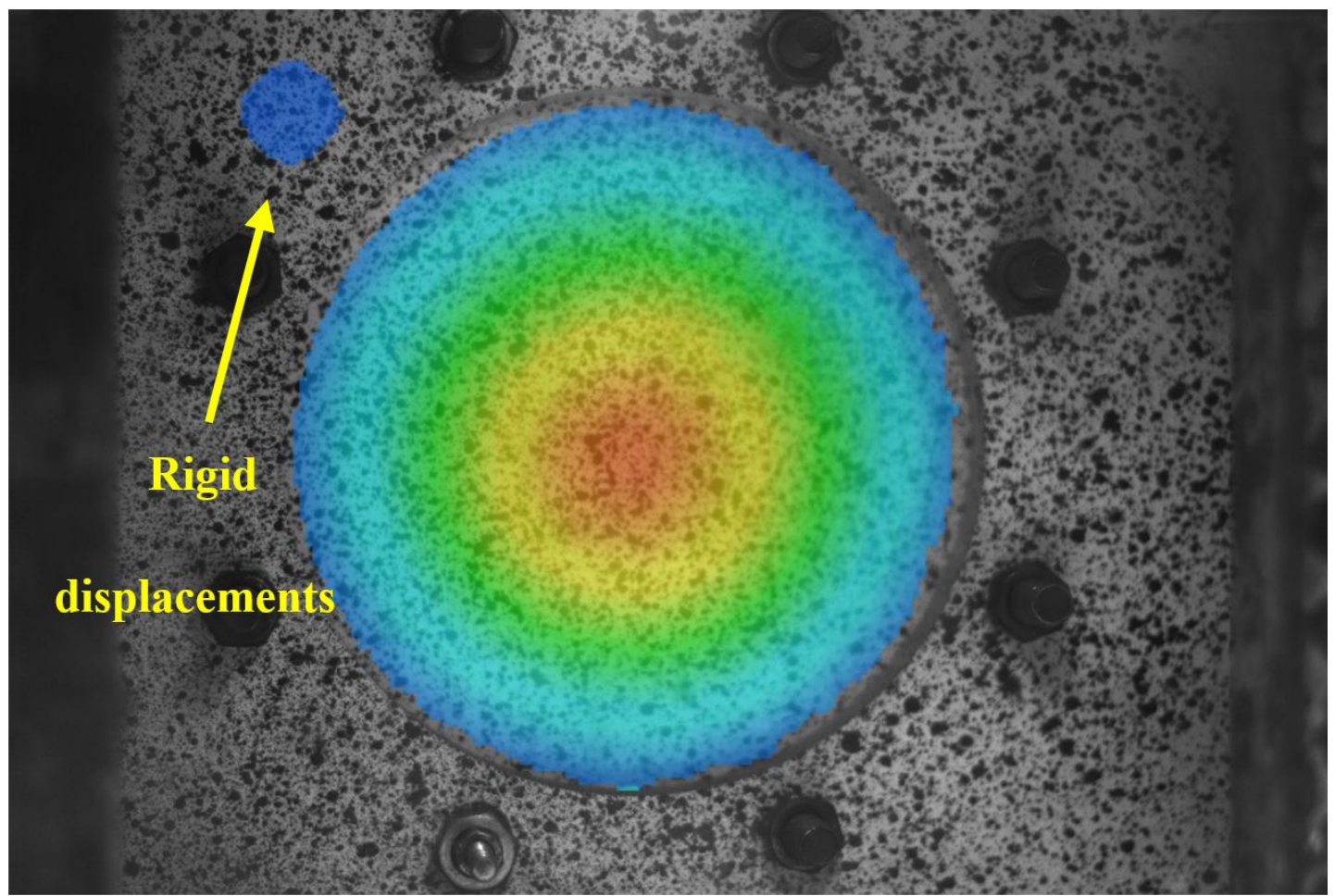

Figure 6. Rigid displacement spot

In order to evaluate the accuracy of DIC results, the comparisons of the deformations between the experiment data and DIC results were made. For the working conditions of $35.7 \mathrm{~m} / \mathrm{s}, 60 \mathrm{~m} / \mathrm{s}$, and $115.6 \mathrm{~m} / \mathrm{s}$, after the impact events were done, the final deformations of the targets were captured and also calculated with DIC. The decision of the impact velocities in this paper were made according to both the scientific and experimental considerations instead of exactly following the real bird-strike situations ranging from 100-300 m/s. On one hand, in bird strike phenomenon, the damage of metal structure is limited to elastic and plastic deformations and no more severe failure modes like penetration or perforation can occur. Therefore, although the size of the projectile and the target used in this paper were different from real background, the working conditions chosen in this paper can cover enough deflection degrees. On the other hand, lower velocities means smaller distortion of the projectiles before impacting on the targets. Figure 7 present the comparisons of deflections of central sections between measured post-test results and DIC results, which shows perfectly consistencies. So the DIC results were reliable for quantitative analysis. Figure 8 shows the post-test pictures of target panels, which were captured with a camera from a perpendicularly lateral direction. From Figure 7 and Figure 8, it can be seen that the post-test deformations of the target panels, or permanent deflections, have a positive correlation with impact velocities. 


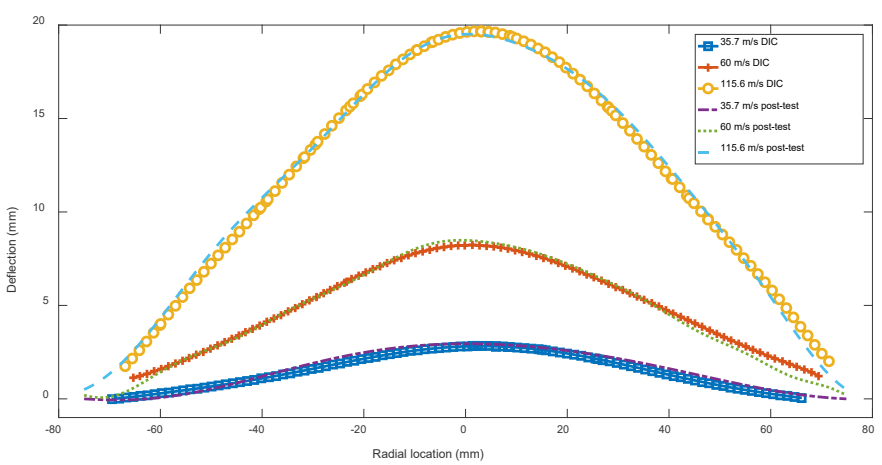

Figure 7. Comparison of deformations of central section between DIC results and post-test results
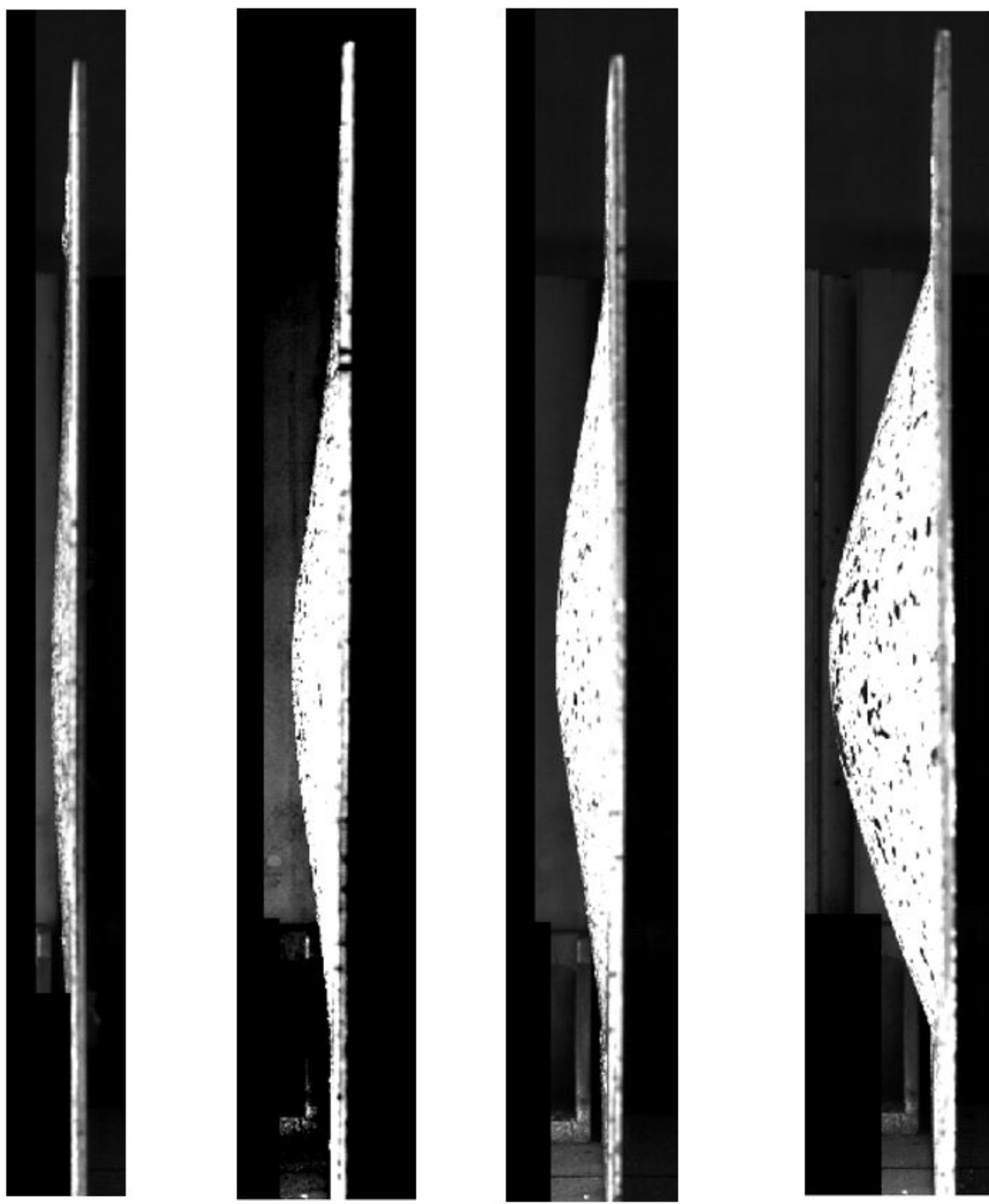

\section{$35.7 \mathrm{~m} / \mathrm{s} \quad 60 \mathrm{~m} / \mathrm{s} \quad 62.9 \mathrm{~m} / \mathrm{s} \quad 115.6 \mathrm{~m} / \mathrm{s}$}

Figure 8. Post-test target panels 
Figure 9 shows a typical plot of out-of-plane displacement together with the images of the projectile during impact event. The colors from dark blue to dark red indicate the scale of z-direction displacements of the particles on the panel from the minimum of $0 \mathrm{~mm}$ to maximum of $10 \mathrm{~mm}$. Since they are much larger than $\mathrm{x}$-direction and $\mathrm{y}$-direction displacements, the surface maps in Figure 9 are approximately the actual shapes of the panel at an interval of $0.2 \mathrm{~ms}$. The light spot at the moment of 0.2 ms indicates the impact zone, from which the bulge was initiated to grow. The circular displacement gradient shows that the response of target was axial symmetric, which is helpful for making assumptions in theoretical analysis.

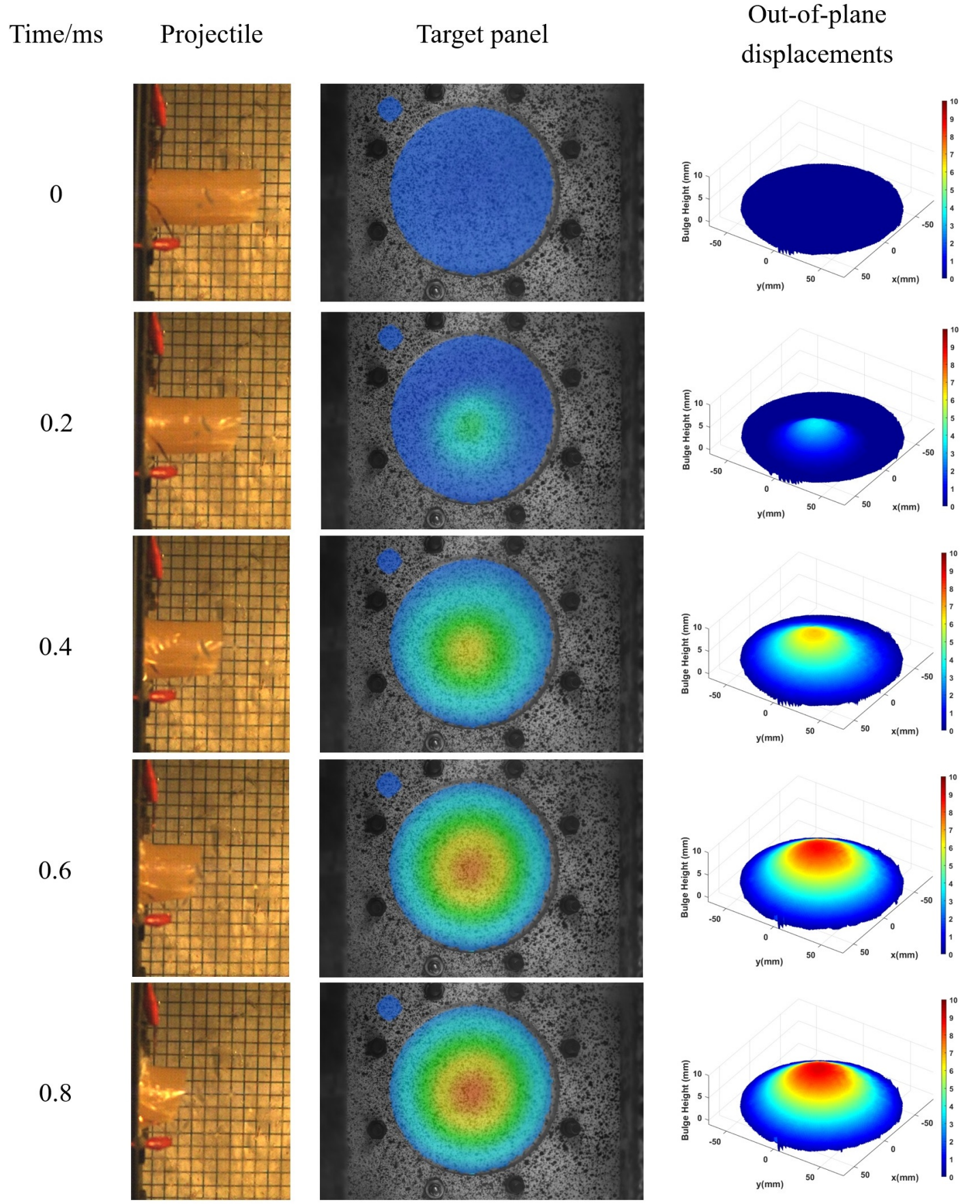

Figure 9. Typical out-of-plane displacement histories $(60 \mathrm{~m} / \mathrm{s}$ impact) 
Figure 10 shows the shape of the central section of the panel at an internal of $0.067 \mathrm{~ms}$. Each line on the plot represents the section's shape at the point in time. The radial location axis, or section coordinates, indicates the location of the particles on the central section ranging from about $-75 \mathrm{~mm}$ to $75 \mathrm{~mm}$, which is basically the loading area's diameter. The time axis indicates the moment when the section line was belonging to. Since the impact duration was $1.16 \mathrm{~ms}$, this figure has contained the whole impact process of this working condition. The growing process of the bulge is clearly showed from its initiation to stable vibration. When the panel was impacted by the projectile, a shock wave was formed and the panel started to deform. Due to the fact that the impact zone was $40 \mathrm{~mm}$ in diameter, a circular plastic hinge was formed th the edge of the zone and flow along the radial direction, as shown in Figure 10. After the initiation of the bulge, the plastic hinge flowed back and forth between the boundary and the center point, while the impact entered the steady flow regime. This can bring in-plane displacements of the particles, but not with a large scale which has been stated above. After the impact energies were all absorbed by the plastic hinges, the bulge reached its final shape and left only elastic vibrations.

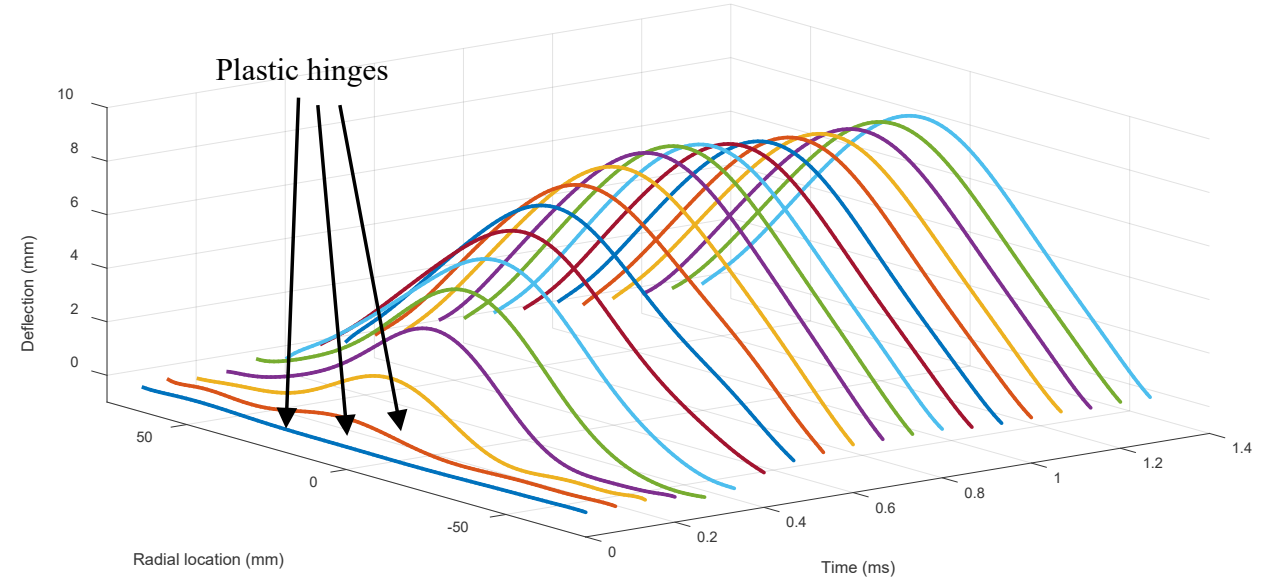

Figure 10. Deformation history of the central section $(60 \mathrm{~m} / \mathrm{s}$ impact)

Figure 11 compares the displacement histories of different points on the target panel subjected to $60 \mathrm{~m} / \mathrm{s}$ impact. Considering the trajectory deviation, the point with the most violent response, or having the largest peak out-of-plane displacement, was set as the center point. The points that were $20 \mathrm{~mm}, 40 \mathrm{~mm}$ and $60 \mathrm{~mm}$ away from the center point were also tracked for comparison. As expected, the farther the points were away from the center, the less peak z-displacement they will reach. Since the projectile had a diameter of $40 \mathrm{~mm}$, the points that was $20 \mathrm{~mm}$ away from the center, which was at the edge of the impact zone, started movement at the same time as the center point. Other two points had to wait for the arrival of the plastic hinge to initiate their displacements. The period between the red vertical line indicate the impact duration calculated using equation (1). For all the points, peak displacements were reached before the impacts were over, which implies that the loadings were not constant. 


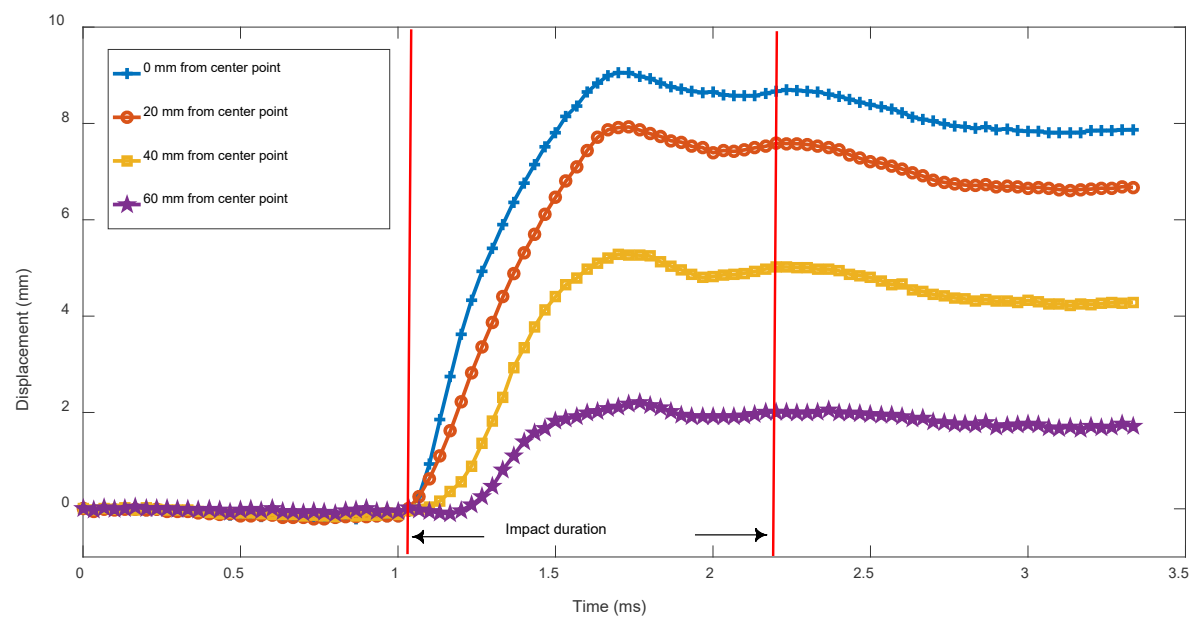

Figure 11. Displacement-Time curves of points on the target panel (60 m/s impact)

The comparisons of peak deflection and deformation histories of the panel under different impact conditions are shown in Figure 12 and Figure 13. Comparison of displacement histories of center points subjected to different impact conditions profiles of peak mid-plane sections shown in Figure 12 indicates the positive correlations between impact energies and magnitudes of deflections. The displacement histories shown in Figure 11 and Figure 13 both imply the deformation process during impact that the panels undertook complicate loading patterns. They got pushed forward and formed the bulges, then bounced back and entered the vibration phases. The curves show good repeatability. Figure 14 displays peak displacements of points on the different location of the panels subjected to different impact conditions. The results are consistent with expectations.

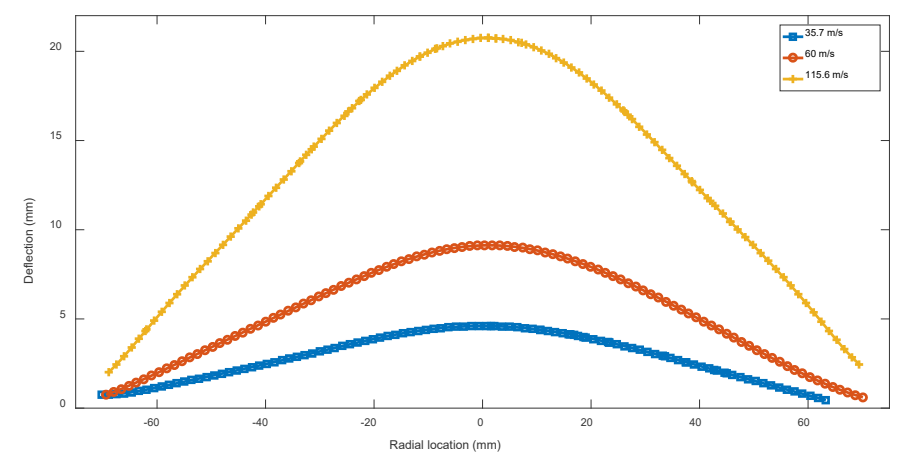

Figure 12. Comparison of peak deflections of panels during impact 


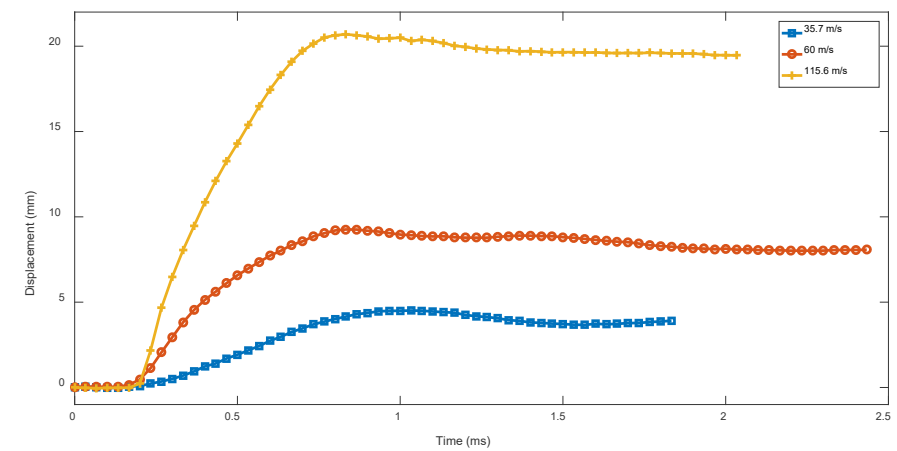

Figure 13. Comparison of displacement histories of center points subjected to different impact conditions

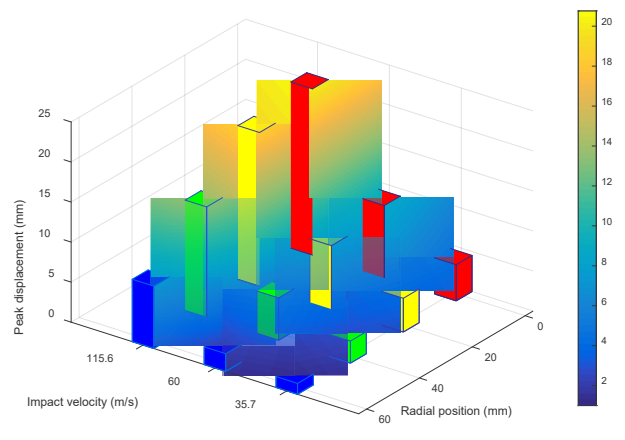

Figure 14. Peak displacements of points on the target panels subjected to different impact conditions

\section{Discussions}

In order to better understand the response of target panels subjected to soft body impact, concentrations would be focused on their energy absorption properties. DIC results has provided abundant data source for deformation observations, which is also helpful to analyze the energy absorption properties of target panels. In the present study, the major fraction of impact energy absorbed by $2 \mathrm{~A} 12$ aluminum panels are plastic work caused by their plastic deformations. The basic energy balance equation can be written as:

$E_{i}=W_{p}+E_{c}+W_{d i s}$

Where $E_{i}, W_{p}$ and $E_{c}$ are respectively the impact energy of projectiles, the plastic work of the panels and the kinetic energy of the panels. $W_{d i s}$ is other kind of energy exchange such as heat dissipations, deformation energy of projectiles and residual kinetic energy of projectiles. Among these items, $W_{d i s}$ is extremely hard to analyze and of less concern in this paper, so it will not be discussed.

\subsection{Plastic work}

The work dissipated by metal panels' plastic deformations is an important quality for impact resistance estimations. In the case of dynamic impact event, the accumulated plastic work of a deformed panel can be calculated using its final shape, or total plastic deformation. Gharababaei and Darvizeh (2010) used the profiles of panels' mid-plane sections to calculate their plastic work. A zero-order Bessel function was used to fit the shapes of the panel bulges. Since the displacement data have all been obtained, they will directly be used without fitting process. 
In order to simplify the model, several assumptions are made, like in Gharababaei and Darvizeh (2010). Firstly, the panels are considered to be perfect rigid plastic material, and the post-impact deformations are all formed by mean plastic strain; Secondly, in-plane displacements of the particles on the panels are ignored, meaning that the radial coordinates are fixed on the space coordinate systems; Thirdly, the deformations of the panels are assumed to be axial symmetric, therefore the plastic work will also be calculated using the profiles of mid-plane sections in this study.

The accumulated plastic work can be calculated by:

$W_{p}=\int_{V}\left(\sigma_{r} \varepsilon_{r}+\sigma_{\theta} \varepsilon_{\theta}\right) d V$

where $\sigma_{r}$ and $\sigma_{\theta}$ are radial and circumferential stress respectively; $\varepsilon_{r}$ and $\varepsilon_{\theta}$ are radial and circumferential strain respectively; $\mathrm{V}$ is the volume of the entire panel. The radial strain, radial and circumferential bending are considered to be the major factors to cause plastic deformations. The shear force and circumferential strain are neglected. The values of strains can be obtained by Gharababaei and Darvizeh (2010):

$\varepsilon_{r}=\varepsilon_{m}+\varepsilon_{r b}=\frac{1}{2}\left(\frac{\partial u}{\partial r}\right)^{2}+z \chi_{r}$

$\varepsilon_{\theta}=\varepsilon_{r b}=z \chi_{\theta}$

where $\varepsilon_{m}, \varepsilon_{r b}$ and $\varepsilon_{\theta b}$ are radial membrane strains, radial bending strains and circumferential bending strains, respectively. $u$ and $r$ are transverse displacements and radial coordinates of particles on the panel, as shown in Figure $15 ; \mathrm{z}$ is the transverse coordinates of the particles ranging from $-\mathrm{H} / 2$ to $\mathrm{H} / 2 ; \quad \chi_{\mathrm{r}}, \quad \chi_{\theta}$ are radial and circumferential curvatures on the panel, which are given by:

$\chi_{r}=-\frac{\partial^{2} u}{\partial r^{2}}$

$\chi_{\theta}=-\frac{1}{r} \frac{\partial u}{\partial r}$

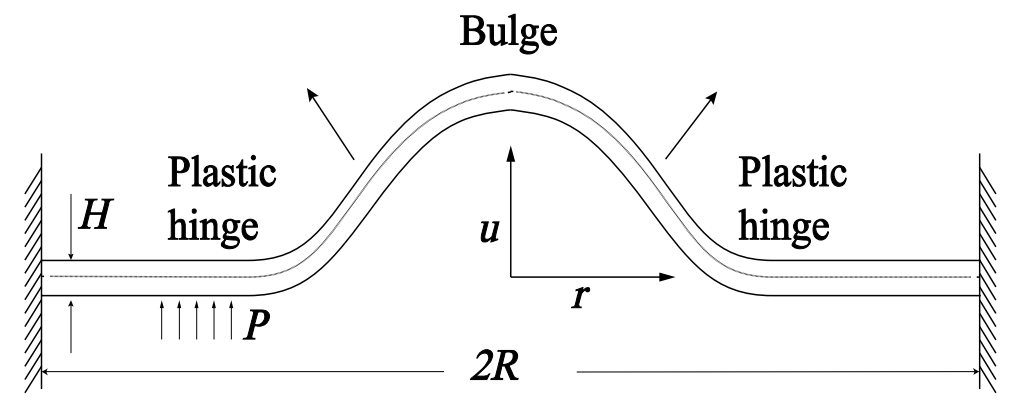

Figure 15. Schematic of panel's bulge 


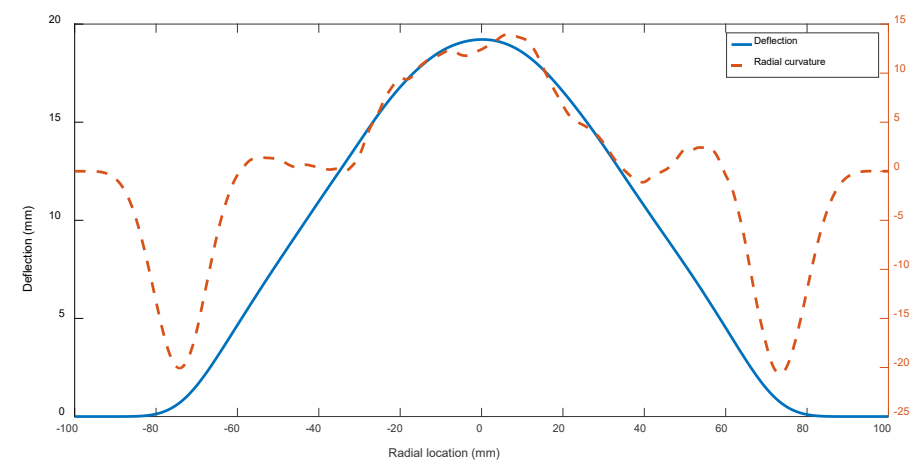

Figure 16. Typical radial curvatures of a post-test panel

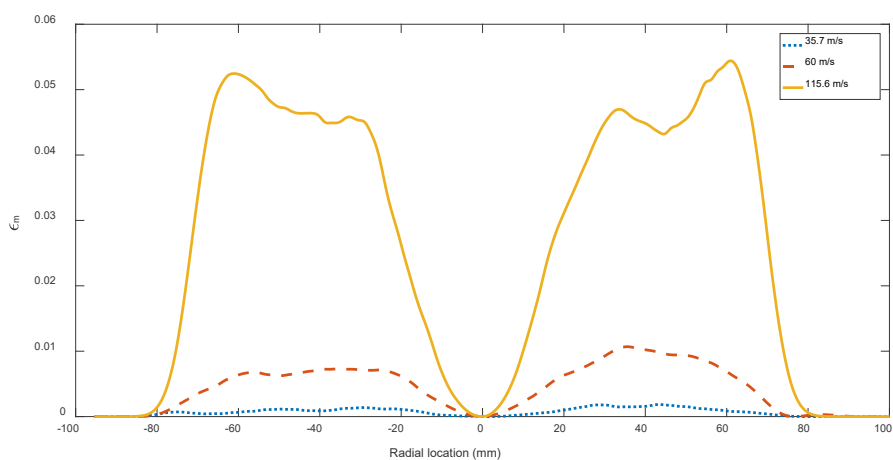

Figure 17. Radial membrane strain distributions on the panels

A typical curve of a post-test panel's mid-plane radial curvatures is shown in Figure 16, with its deflection profile as a reference. As expected, the largest plastic bending deformations happened on the center and the bounding edge of the panel. Figure 17 displays the radial membrane strain distributions on the panels subjected to different loading conditions. It can be seen that the major plastic strains occurred on the annular regions between the center points and the bounding edges. Larger impact force resulted in larger radial deformations.

The radial and circumferential stress can be substituted by the mean dynamic flow stress based on the assumption of rigid plastic material and flow relation:

$\sigma_{r}=\sigma_{\theta}=\sigma_{d}$

$\sigma_{d}=\sigma_{y}\left[1+\left(\frac{\dot{\varepsilon}_{m}}{D}\right)^{\frac{1}{q}}\right]$

where $\dot{\varepsilon}_{m}$ represents for the mean strain rate; D and q are constant parameters which are 6500 and 4 respectively for aluminum alloy; $\sigma_{y}$ is the yield stress; $\sigma_{d}$ is the mean dynamic flow stress determined by the Cowper-Symonds empirical equation. Substituting Equation (5)-(10) into Equation (4) yields:

$W_{m}=2 \pi \sigma_{d} \int_{0}^{R} \int_{-H / 2}^{H / 2}\left(\varepsilon_{m}\right) r d z d r=2 \pi \sigma_{d} \int_{0}^{R}\left[\frac{1}{2}\left(\frac{\partial u}{\partial r}\right)^{2} H r\right] d r$ 


$$
\begin{aligned}
& W_{\chi_{r}}=2 \pi \sigma_{d} \int_{0}^{R} \int_{-H / 2}^{H / 2}\left(\varepsilon_{r b}\right) r d z d r=2 \pi \sigma_{d}\left[\frac{1}{4} H^{2} \int_{0}^{R}\left(-\frac{\partial^{2} u}{\partial r^{2}} r\right) d r\right] \\
& W_{\chi_{\theta}}=2 \pi \sigma_{d} \int_{0}^{R} \int_{-H / 2}^{H / 2}\left(\varepsilon_{r \theta}\right) r d z d r=2 \pi \sigma_{d}\left[\frac{1}{4} H^{2} \int_{0}^{R}\left(-\frac{\partial u}{\partial r}\right) d r\right]
\end{aligned}
$$

and

$$
W_{p}=W_{m}+W_{\chi_{r}}+W_{\chi_{\theta}}
$$

where $W_{m}, W_{\chi_{r}}$ and $W_{\chi_{\theta}}$ are plastic work components done by radial membrane stress, radial and circumferential bending moments, respectively. From Equation (11)-(13) we can see that the plastic work of a deformed panel can be calculated using only the deflection data of the particles on it, which are available in DIC results. The differentials were calculated using numerical gradients in the MATLAB software. Before the calculations, the Piecewise Cubic Hermite Interpolating Polynomial (PCHIP) method along with the moving average filter were used on the displacement data in order to obtain more smoothed results. As for the integral calculations, the trapezoidal numerical integration method was used. For example, in order to calculate $\int_{0}^{R} f(r) d r$, where $f(r)=\frac{1}{2}\left(\frac{\partial u}{\partial r}\right)^{2} H r$, in Equation (11), the integral can be approximated to:

$$
\int_{0}^{R} f(r) d r \approx \frac{1}{2} \sum_{n=1}^{N}\left(r_{n+1}-r_{n}\right)\left[f\left(r_{n+1}\right)+f\left(r_{n}\right)\right]
$$

where the subscripts represent for the spaced points which were obtained in the interpolating process. Dimensionless methods are then used to quantify the data:

$$
\left\{\begin{array}{l}
\bar{W}_{p}=W_{p} / E_{i} \\
\bar{W}_{m}=W_{m} / E_{i} \\
\bar{W}_{x_{r}}=W_{x_{r}} / E_{i} \\
\bar{W}_{x_{\theta}}=W_{x_{\theta}} / E_{i}
\end{array}\right.
$$

where $E_{i}$ is the impact energy brought by the projectile.

Figure 18(a) displays the comparison of dimensionless plastic work $\bar{W}_{p}$ under different impact loading conditions. When the impact energy was $63.72 \mathrm{~J}$, the energy absorbed by plastic deformation only occupied $43.9 \%$ of the total work. Other dissipation forms like heat conductions and projectile deformations consumed most of the energies. But these dissipation channels have limitations. When the impact energies were increased to $668.2 \mathrm{~J}$, the plastic deformations 
became the major energy dissipation form (98.9\%). The components of the dimensionless plastic work $\left(\bar{W}_{m}, \quad \bar{W}_{x_{r}}, \quad \bar{W}_{x_{\theta}}\right.$ ) are compared in Figure 18(b). It can be seen that the plastic work done by radial membrane stress were the main fractions of energy absorptions. However, when the impact energy was low (63.72 J), the radial bending moment consumed nearly the same level of energy as radial membrane stress. Increasing the impact loadings decreased the energy consumptions of radial bending. The energies dissipated by circumferential bending are very small compared with other two forms.

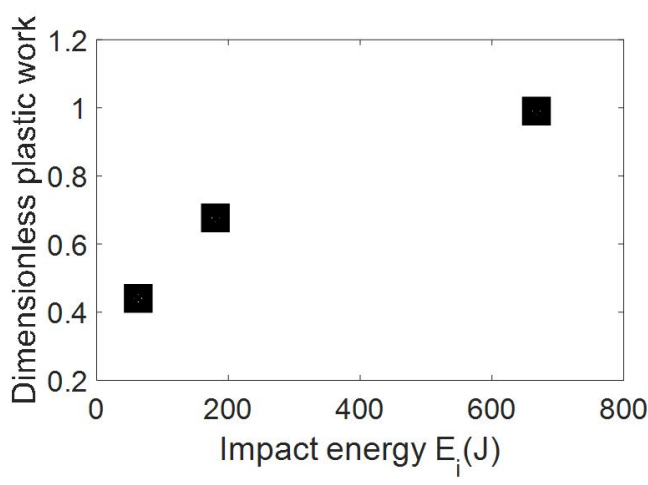

(a)

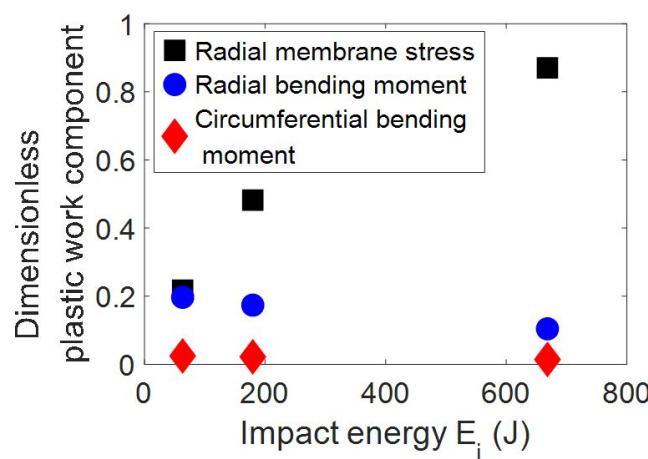

(b)

Figure 18. Comparison of dimensionless plastic work. (a) dimensionless plastic work under different loading conditions, (b) dimensionless plastic work components under different loading conditions

\subsection{Time-dependent energy exchange}

The dynamic response of the target panel can give us a deeper insight into the energy exchange process. Since the profiles of bulges of every picture can be obtained, the dynamic deformation histories of the panels are available for this issue. Figure 19 shows the time-dependent radial membrane strain of the mid-plane section, with each line represents for the strain profile at the point in time, which are obtained by equation (5). These data, along with other dynamic data, can be used to calculate the kinetic energies and dynamic plastic work in the whole impact process.

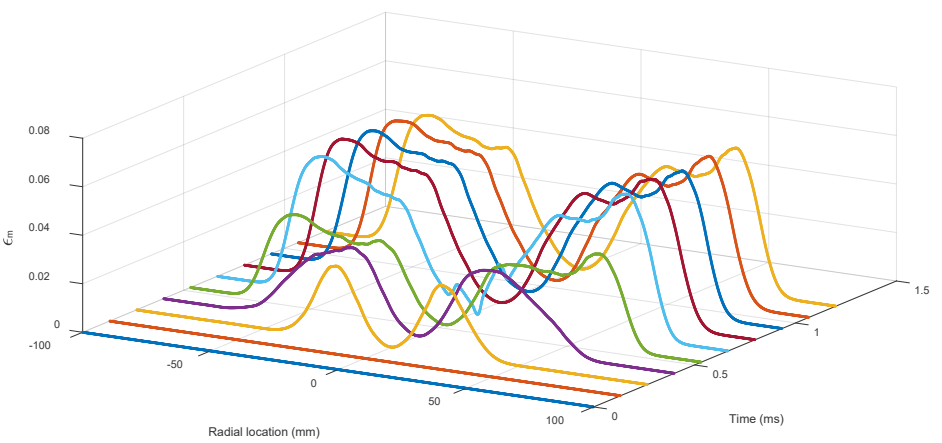

Figure 19. Typical radial membrane strain of the mid-plane section during impact

R. Zaera Zaera et al. (2002) provided an analytical model for panels subjected to a transverse impulsive force. He used the rates of work to describe the time-dependent work balance. In his model, the kinetic energies of the panel can be divided into translation kinetic energies and rotatory kinetic energies: 


$$
\left\{\begin{array}{l}
d E_{c}^{t}=\frac{1}{2} d m\left(\frac{d u}{d t}\right)^{2} \\
d E_{c}^{r}=\frac{1}{2} d I_{\theta} \dot{\theta}^{2}=\arctan \left(\frac{\partial u}{\partial r}\right)
\end{array}\right.
$$

where $d E_{c}^{t}$ and $d E_{c}^{r}$ are elemental translation and rotatory kinetic energies respectively; $d m$ and $d I_{\theta}$ are elemental mass and moment of inertia about the circumferential axis; $\dot{\theta}$ is the angular speed. The total kinetic energies can be given by:

$$
E_{c}=E_{c}^{t}+E_{c}^{r}
$$

The dimensionless kinetic energies and time are:

$$
\left\{\begin{array}{l}
\bar{E}_{c}=E_{c} / E_{i} \\
\bar{E}_{c}^{t}=E_{c}^{t} / E_{i} \\
\bar{E}_{c}^{r}=E_{c}^{r} / E_{i} \\
\bar{t}=t / t_{\text {final }}
\end{array}\right.
$$

Figure 20(a) compares the total kinetic energies in the impact durations. Based on the energy balance relations, when the targets were interacting with the projectiles, the energy exchange had started immediately. The kinetic energies were transferred from the impact event, and the target panels' deformations were initiated accompanied with elastic potential energies and following plastic work. After reaching their peak values, the kinetic energies decreased and major of them were transferred to other type of work. For the three impact conditions, the kinetic energies occupied only less than $10 \%$ of the total energies. With higher impact velocities, the kinetic energies reached higher peak values. Figure 20(b) shows the ratio of transverse and rotatory kinetic energies. It can be seen that during the whole impact process, the kinetic energies brought by transverse displacement were much larger than rotatory inertia. This is similar to R. Zaera's results although the loading conditions were different. 


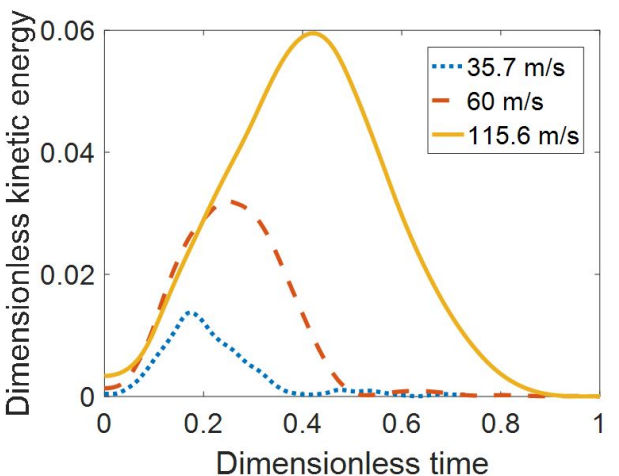

(a)

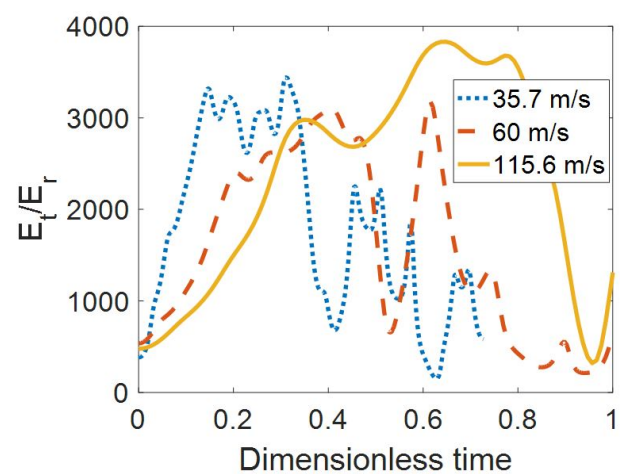

(b)

Figure 20. Comparison of time-dependent kinetic energies. (a) Dimensionless total kinetic energies, (b) The ratio of transverse and rotatory kinetic energies

The rate of panels' plastic work can be obtained by differentiating equation (14) with respect to time, and consequently the history curves of their accumulated plastic work can be plotted. However, the major error will occur when using the assumption of rigid plastic material properties to describe the response of panels under dynamic impact loadings. As seen from Figure 11, the panels had remarkable elastic vibrations after reaching their peak deflections, proving that the elastic deformations cannot be ignored. The rigid plastic assumptions will replace the elastic state of strain with plastic flow strain, which will enlarge the stress during impact. Furthermore, the flow of plastic hinges will make the state of stress extremely hard to analyze. Figure 21(a) plots a typical time-dependent curve of panel's plastic work, which surpassed the initial impact energy during impact. Also, after the deflection began to vibrate, the plastic work was still increasing. This is because the elastic vibrations were calculated as plastic flow, therefore the plastic work was overestimated. The comparison of obtained plastic work is shown in Figure 21(b), where a negative correlation between the dimensionless plastic work and impact velocities can be figured out. This is due to the fact that elastic deformations have their limitations. When the impact loadings were increased, the plastic work became the major way for the panels' energy absorptions. It can be seen that the dimensionless plastic work of the panel under $115.6 \mathrm{~m} / \mathrm{s}$ impact reached nearly 1 finally, meaning that the fraction of overestimated work was less than those under smaller loading conditions.

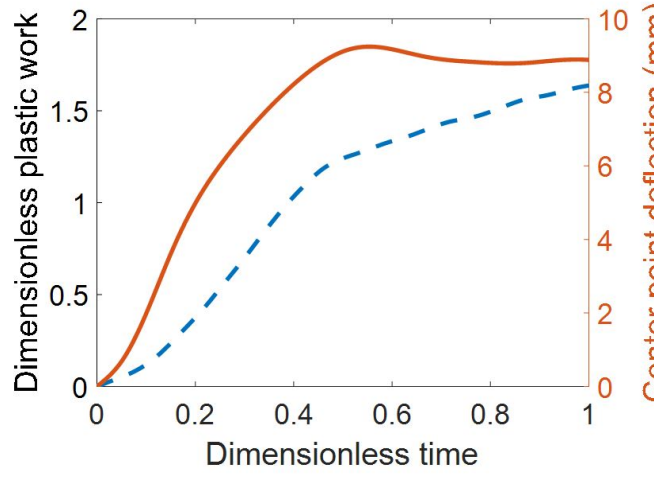

(a)

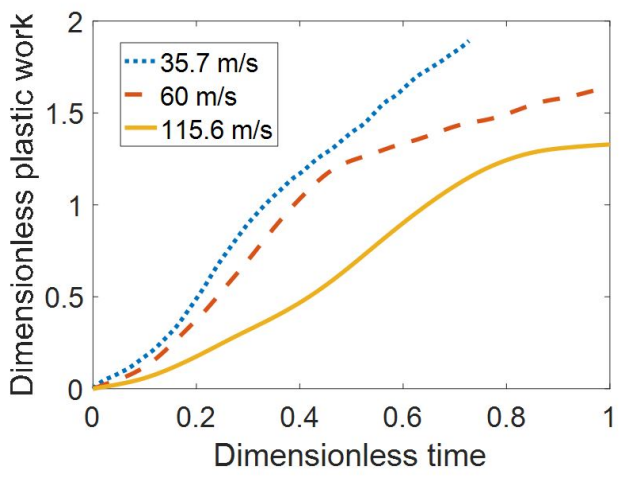

(b)

Figure 21. Comparison of dimensionless time-dependent plastic work. (a) typical history curve of plastic work and corresponding center point deflection, (b) comparison of time-dependent plastic work under different loading conditions 


\section{Concluding remarks}

Soft body impact issues are widely concerned in today's researches. This paper employed DIC technique to obtain the 3D deformation process of aluminum targets subjected to gelatin impact experiments. With carefully designed experiments and evaluations of DIC results, reliable time-dependent experimental data were collected and regulations were analyzed. The in-plane displacements on the panels were negligible compared to out-of-plane deflections, therefore the following discussion were based on transverse deflections of the panels. The loading area basically satisfy the axial symmetric assumptions, which was helpful for theoretical analysis. Dynamic plastic flow and elastic vibrations occurred in the impact process observed from the deformation histories of points and mid-plane sections on the panels, which made the state of stress extremely hard to determined.

Energy absorption properties of the target panels were theoretically discussed using energy balance equations. The post-test shapes of the panels were used to calculate the accumulated work absorbed by plastic deformations of the panels. With the impact energies increased, plastic work became the major channel for energy consumptions. The radial membrane forces were found to be the main fraction for the dissipation of impact energies compared with radial and circumferential bending moments. The time-dependent energy exchange can be obtained by differentiating the work balance equations. Since the elastic vibrations during impact had limitations, the fraction of accumulated plastic work from total impact energies had negative correlation with impact velocities. However, the major error existed in the plastic work calculations that the rigid plastic assumptions were inappropriate. So the time-dependent plastic work calculated by the model in this paper were overestimated. In future work, more experimental researches are required for energy balance model evaluations. More accurate models of elastic-plastic material are needed to better calculations of timedependent energy absorption process of impact events.

Author's Contributions: Conceptualization, D Li and W Zhang; Data curation, D Li; Formal analysis, D Li; Investigation, D Li, W Xie and X Jiang; Methodology, D Li and W Zhang; Resources, W Zhang and L Guo; Software, D Li; Supervision, W Zhang; Validation, D Li, W Zhang and L Guo; Visualization, D Li; Writing - original draft, D Li; Writing - review \& editing, D Li.

Editor: Marcílio Alves.

\section{References}

Airoldi A., Cacchione B. (2006). Modelling of impact forces and pressures in Lagrangian bird strike analyses. International Journal of Impact Engineering 32:1651-1677.

Anghileri M., Milanese A., Moretti G., Castelletti L. (2012). Preliminary investigation on the feasibility of a bird surrogate for fullscale bird impact test 3:2144-2151.

Bhatnagar N. (2014). Digital Image Correlation of Low-Velocity Impact on a Glass/Epoxy Composite. International Journal for Computational Methods in Engineering Science \& Mechanics 15:203-217.

Chen W., Song B. (2011) Split Hopkinson (Kolsky) Bar Springer US.

Chu T.C., Ranson W.F., Sutton M.A. (1985). Applications of digital-image-correlation techniques to experimental mechanics. Experimental Mechanics 25:232-244. 
Corporation H.P. (2012). High-Velocity Impact Behaviour of Prestressed Composite Plates under Bird Strike Loading. International Journal of Aerospace Engineering 2012.

Flores M., Mollenhauer D., Runatunga V., Beberniss T., Rapking D., Pankow M. (2017). High-speed 3D digital image correlation of low-velocity impacts on composite plates. Composites Part B: Engineering 131:153-164.

Gharababaei H., Darvizeh A. (2010). Experimental and Analytical Investigation of Large Deformation of Thin Circular Plates Subjected to Localized and Uniform Impulsive Loading. Mechanics Based Design of Structures and Machines 38:171-189.

Hanssen A.G., Girard Y., Olovsson L., Berstad T., Langseth M. (2006). A numerical model for bird strike of aluminium foambased sandwich panels. International Journal of Impact Engineering 32:1127-1144.

Heimbs S. (2011). Computational methods for bird strike simulations: A review. Computers \& Structures 89:2093-2112.

Heimbs S., Bergmann T. (2012). High-Velocity Impact Behaviour of Prestressed Composite Plates under Bird Strike Loading. International Journal of Aerospace Engineering,2012,(2012-4-19) 2012:11 pages.

Hild F., Bouterf A., Roux S. (2019). Measurement of kinematic fields via DIC for impact engineering applications. International Journal of Impact Engineering 130:163-171.

Huang J., Guo Y., Qin D., Zhou Z., Li D., Li Y. (2018). Influence of stress triaxiality on the failure behavior of Ti-6Al-4V alloy under a broad range of strain rates. Theoretical and Applied Fracture Mechanics 97:48-61.

Kumar P., LeBlanc J., Stargel D.S., Shukla A. (2012). Effect of plate curvature on blast response of aluminum panels. International Journal of Impact Engineering 46:74-85.

Lall P., Shantaram S., Angral A., Kulkarni M. (2009) Explicit submodeling and digital image correlation based life-prediction of leadfree electronics under shock-impact, Electronic Components \& Technology Conference.

Lavoie M.A., Gakwaya A., Ensan M.N., Zimcik D.G. (2007a) Review of existing numerical methods and validation procedure available for bird strike modeling.

Lavoie M.A., Gakwaya A., Ensan M.N., Zimcik D.G. (2007b). Validation of available approaches for numerical bird strike modeling tools. International Review of Mechanical Engineering 1:380-389.

Lavoie M.A., Gakwaya A., Ensan M.N., Zimcik D.G., Nandlall D. (2009). Bird's substitute tests results and evaluation of available numerical methods. International Journal of Impact Engineering 36:1276-1287.

Liu J., Li Y., Gao X. (2014). Bird Strike on a Flat Plate: Experiments and Numerical Simulations. International Journal of Impact Engineering 70:21-37.

Mao R., Meguid S.A., Teng Y.N. (2007). Finite Element Modeling of a Bird Striking an Engine Fan Blade. Journal of Aircraft 44:583-596.

Mao R.H., Meguid S.A., Ng T.Y. (2008). Transient three dimensional finite element analysis of a bird striking a fan blade. International Journal of Mechanics \& Materials in Design 4:79-96.

Mao R.H., Meguid S.A., Ng T.Y. (2009). Effects of incidence angle in bird strike on integrity of aero-engine fan blade. International Journal of Crashworthiness 14:295-308.

Meguid S.A., Mao R.H., Ng T.Y. (2008a). FE analysis of geometry effects of an artificial bird striking an aeroengine fan blade. International Journal of Impact Engineering 35:487-498. 
Meguid S.A., Mao R.H., Ng T.Y. (2008b). FE analysis of geometry effects of an artificial bird striking an aeroengine fan blade. International Journal of Impact Engineering 35:487-498.

Nishikawa M., Hemmi K., Takeda N. (2011). Finite-element simulation for modeling composite plates subjected to soft-body, high-velocity impact for application to bird-strike problem of composite fan blades. Composite Structures 93:1416-1423.

Seidt J.D., Pereira J.M., Hammer J.T., Gilat A. (2012) Dynamic Load Measurement of Ballistic Gelatin Impact Using an Instrumented Tube, XII International Congress \& Exposition on Experimental \& Applied Mechanics. pp. 243-250.

Sutton M.A., Mingqi C., Peters W.H., Chao Y.J., McNeill S.R. (1986). Application of an optimized digital correlation method to planar deformation analysis. Image and Vision Computing 4:143-150.

Sutton M.A., Wolters W.J., Peters W.H., Ranson W.F., McNeill S.R. (1983). Determination of displacements using an improved digital correlation method. Image and Vision Computing 1:133-139.

Tang Z., Hang C., Suo T., Wang Y., Dai L., Zhang Y., Li Y. (2017). Numerical and experimental investigation on hail impact on composite panels. International Journal of Impact Engineering 105:102-108.

Tiwari V., Sutton M.A., McNeill S.R., Xu S., Deng X., Fourney W.L., Bretall D. (2009). Application of 3D image correlation for fullfield transient plate deformation measurements during blast loading. International Journal of Impact Engineering 36:862-874. Tuo H., Lu Z., Ma X., Zhang C., Chen S. (2019). An experimental and numerical investigation on low-velocity impact damage and compression-after-impact behavior of composite laminates. Composites Part B: Engineering 167:329-341.

Wilbeck J.S. (1978). Impact behavior of low strength projectiles.

Wilbeck J.S., Rand J.L. (1981). The Development of a Substitute Bird Model. Journal of Engineering for Gas Turbines \& Power 1:725-730.

Yuan Y., Wang S. (2019). Measurement of the energy release rate of compressive failure in composites by combining infrared thermography and digital image correlation. Composites Part A: Applied Science and Manufacturing 122:59-66.

Zaera R., Arias A., Navarro C. (2002). Analytical modelling of metallic circular plates subjected to impulsive loads. International Journal of Solids \& Structures 39:659-672.

Zhang P., Porfiri M. (2019). A combined digital image correlation/particle image velocimetry study of water-backed impact. Composite Structures 224:111010.

Zhang W., WEI G., XIAO X.-k. (2013). Constitutive Relation and Fracture Criterion of 2A12 Aluminum Alloy. Acta Armamentarii 3:003. 